\title{
Reactivity of Alkyldibenzothiophenes Using Theoretical Descriptors
}

\author{
Jose Luis Rivera, ${ }^{1}$ Pedro Navarro-Santos, ${ }^{2}$ Luis Hernandez-Gonzalez, ${ }^{1}$ \\ and Roberto Guerra-Gonzalez ${ }^{1}$ \\ ${ }^{1}$ Facultad de Ingeniería Química, Universidad Michoacana de San Nicolás de Hidalgo, 58010 Morelia, MICH, Mexico \\ ${ }^{2}$ Facultad de Tecnología de la Madera, Universidad Michoacana de San Nicolás de Hidalgo, 58010 Morelia, MICH, Mexico
}

Correspondence should be addressed to Jose Luis Rivera; jlrivera@umich.mx

Received 4 November 2013; Accepted 11 February 2014; Published 26 March 2014

Academic Editor: Natarajan S. Venkataramanan

Copyright (C) 2014 Jose Luis Rivera et al. This is an open access article distributed under the Creative Commons Attribution License, which permits unrestricted use, distribution, and reproduction in any medium, provided the original work is properly cited.

\begin{abstract}
Theoretical calculations of the reactivity of dibenzothiophene and its methyl, dimethyl, and trimethyl derivatives show that local reactivity descriptors reproduce their experimental desulfurization reactivity trend if the first desulfurization step involves directly the sulfur atom, which only occurs if the sulfur atom is blocked at most by one methyl group. In the series of molecules $\{4,7$-dimethyldibenzothiophene, $x, 4,7$-trimethyldibenzothiophene $(x=1,2,3)\}$, the most reactive molecule is $2,4,7-$ trimethyldibenzothiophene, and local descriptors show that the reactivity is linked to the activity of the sulfur atom, which is higher in 2,4,7-trimethyldibenzothiophene due to the position of the third methyl substitute, located in the para position with respect to the carbon bonded to the sulfur atom. The electrostatic potential of 2,4,7-trimethyldibenzothiophene shows one effective adsorption site, while 1,4,7-trimethyldibenzothiophene and 3,4,7-trimethyldibenzothiophene have more sites, contributing to the higher reactivity of 2,4,7-trimethyldibenzothiophene. The index of reactivity of other descriptors was evaluated and the effect of the position of the methyl substituents on adsorption parameters, as the dipole moment and the atomic charges were also studied.
\end{abstract}

\section{Introduction}

Desulfurization processes of refractory organosulfur compounds such as 4-methyldibenzothiophene (4-MDBT) and 4,6-dimethyldibenzothiophene (4,6-DMDBT) and their alkyl derivatives show major technological and scientific challenges. To transform these molecules through direct desulfurization, the organosulfur molecules should adsorb through the sulfur atom oriented perpendicularly to the catalytic surface $[1,2]$. The refractory organosulfur molecules exhibit steric effects on their sulfur atom by one or two methyl substitutes on the aromatic rings that make these molecules very difficult to adsorb with the appropriate orientation to the catalytic surfaces. The level of hindrance in the sulfur atom is not the only factor involved in the desulfurization of these molecules, because, even for molecules that show the same level of hindrance in their sulfur atom, its experimental reactivity exhibits different behavior [3-5]. For example, studies of desulfurization of organosulfur molecules in the group 1,4,7-trimethyldibenzothiophene (1,4,7-TMDBT), 2,4,7-trimethyldibenzothiophene (2,4,7-TMDBT), and 3,4,7trimethyldibenzothiophene (3,4,7-TMDBT) show that 2,4,7TMDBT has a rate constant two times higher than 1,4,7TMDBT and 3,4,7-TMDBT, which are classified as low reactive compounds, as the refractory molecule 4,6-DMDBT [3]. Figure 1 shows a scheme of the series of molecules $\{x, 4,7$ TMDBT, $(x=1,2,3)\}$. The position of the third methyl in 2,4,7-TMDBT is in the para position (with respect to the carbon atom bonded to the sulfur atom), and that position can explain the higher reactivity of 2,4,7-TMDBT, but the position of the third methyl cannot explain the differences observed in the other two molecules, which have their third methyl in the meta position.

There are several computational chemistry studies in the literature dealing with physical $[6,7]$ and chemical properties $[8,9]$ of organosulfur compounds. Studies of adsorption and reactivity of organosulfur molecules on catalytic surfaces using Density Functional Theory (DFT) methodologies have gained insight into the molecular phenomena associated with 


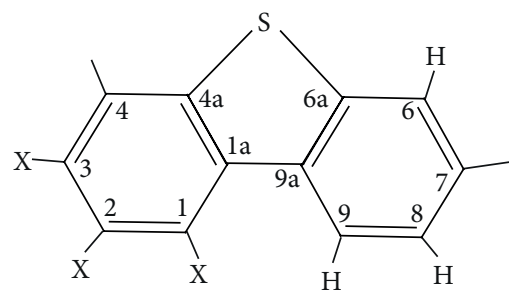

FIGURE 1: Scheme of the studied molecules in the series of molecules $\{x, 4,7-T M D B T,(x=1,2,3)\}: \mathrm{X}(\mathrm{H})-\mathrm{C}_{1}<, \mathrm{X}(\mathrm{H})-\mathrm{C}_{2}<$, and $\mathrm{X}(\mathrm{H})-$ $\mathrm{C}_{3}<$ represent 4,7-DMDBT; $\mathrm{X}\left(\mathrm{CH}_{3}\right)-\mathrm{C}_{1}<, \mathrm{X}(\mathrm{H})-\mathrm{C}_{2}<$, and $\mathrm{X}(\mathrm{H})-$ $\mathrm{C}_{3}<$ represent 1,4,7-TMDBT; $\mathrm{X}(\mathrm{H})-\mathrm{C}_{1}<, \mathrm{X}\left(\mathrm{CH}_{3}\right)-\mathrm{C}_{2}<$, and $\mathrm{X}(\mathrm{H})-$ $\mathrm{C}_{3}<$ represent 2,4,7-TMDBT; $\mathrm{X}(\mathrm{H})-\mathrm{C}_{1}<, \mathrm{X}(\mathrm{H})-\mathrm{C}_{2}<$, and $\mathrm{X}\left(\mathrm{CH}_{3}\right)-$ $\mathrm{C}_{3}<$ represent 3,4,7-TMDBT.

these processes [10-12]. These calculations allow us to predict the active sites for adsorption and chemisorption on specific catalytic surfaces, but due to computation limitations, the size of the studied systems only contains at the most a few hundred atoms. Reactivity descriptors predict the reactivity behavior of molecules based only on the changes that occur within the molecule, without the need to study the whole reaction system $[13,14]$. The reactivity descriptors are also used to predict the reactivity trend in a group of molecules for specific reaction attacks [15-17].

In this paper, we study the reactivity trend in the series of molecules named DBT, $\{x$-MDBT, $(x=3,4)\},\{x, 6$-DMDBT, $(x=3,4)\}, 3,7-\mathrm{DMDBT},\{x, 3,6$-TMDBT, $(x=1,2)\}$, $\{x, 3,7-\mathrm{TMDBT},(x=1,2)\},\{x, 4,6$-TMDBT, $(x=1,2,3)\}$, and $\{x, 4,7-\mathrm{TMDBT},(x=1,2,3)\}$, where dibenzothiophene is abbreviated to DBT, methyldibenzothiophene to MDBT, dimethyldibenzothiophene to DMDBT, and trimethyldibenzothiophene to TMDBT. The reactivity is studied through local descriptors, global descriptors, and the molecular electrostatic potential (MEP) to elucidate the methyl positions which facilitate the desulfurization process. We find that local descriptors reproduce the experimental reactivity trend in the group of molecules $\{x, 4,7$-TMDBT, $(x=1,2,3)\}$ and global descriptors also predict the molecule with the highest reactivity $(2,4,7-\mathrm{TMDBT})$ within the group of studied molecules. MEP maps show that steric effects make one of the two competitive sites of 2,4,7-TMDBT inaccessible and make the sulfur atom more accessible for the adsorption process, contributing to the higher experimental reactivity of 2,4,7TMDBT. Predictions of the highest reactivity correspond to the molecule 3,4,6-TMDBT, but all molecules with substitutes in both $\mathrm{C}_{4}$ and $\mathrm{C}_{6}$ show very similar experimental values, probably indicating that even though the sulfur atom is very reactive, the level of hindrance blocks its real reactivity in the desulfurization processes. This paper is organized as follows. We report the computational methodology and the equations we use to predict the molecular and reactivity properties in Section 2. The results and discussion part of the paper show results for geometrical, electrostatic, and energetic properties of all studied molecules in Section 3. We also show the MEP maps for the studied molecules and report the values of the reactivity descriptors and the predicted trend in reactivity for all series of molecules studied. Finally, we present the conclusions at the end of the paper.

\section{Computational Methods}

We obtain the most stable conformations of all molecules through energy minimizations using DFT calculations and the GAUSSIAN software [18] at the B3LYP/6-311+G(d,p) level of theory. This method and basis set has been employed to predict successfully the proton affinity values of relevant sulfur compounds, with average deviations of $\sim 1 \mathrm{kcal} / \mathrm{mol}$ [19]. We use the following geometry optimization convergence thresholds: 0.00045 Hartrees/Bohr or Hartrees/radians (maximum force), 0.00030 a.u. (root mean square force), 0.0018 Bohr (maximum displacement), and 0.0012 a.u. (root mean square displacement). We carry out vibrational analyses to obtain the vibrational frequencies and verify that the optimized geometries correspond to global minimums. We calculate partial charges and dipole moments using the MerzKollman population analysis [20].

The reactivity of a molecule can be predicted using the Fukui function. This function does not need to study all reactants and catalysts that participate in a reaction but only the objective molecule. It is based on the principle that, during a reaction, there is an exchange of charge between reactants, which produces charge rearrangements within each reactant. The regions of each molecule that suffer more charge rearrangements are the most reactive sites. Then, this methodology compares the charge rearrangement within the molecule when one electron is added to or subtracted from the charge distribution in the ground state and predicts which regions suffer more changes. The condensed Fukui function [21-24] is as follows:

$$
f_{k}^{-}=q_{k}(N)-q_{k}(N-1)
$$

which is related to the electrophilic attack. $q_{k}$ is the partial charge of atom $k . N$ and $N-1$ indicate evaluations of the partial charges at the ground state and the corresponding cationic states, evaluated at the ground conformation.

In the present work, we use the electrostatic potential maps, electronegativity $(\chi)$, global softness (S), and condensed atomic softness $(s)$ to study the reactivity of the molecules. According to Koopmans' frontier orbital theorem [25], $\chi$ can be approximately defined by the following:

$$
\chi=\frac{\mathrm{IP}+\mathrm{EA}}{2},
$$

where IP and EA are the vertical ionization potential and the vertical electron affinity energies, respectively. Similarly, S is approximated by the following:

$$
S=\frac{1}{I P-E A}
$$

while $s$ is calculated by using the following [21]:

$$
s_{k}^{-}=\mathrm{S} f_{k}^{-} \text {. }
$$


TABLE 1: Electrostatic and geometric properties at the ground state for DBT and its methyl, dimethyl, and trimethyl derivatives, calculated using the DFT, the B3LYP/6-311+G(d,p) basis set, and the Merz-Kollman population analysis [20]: charge of the sulfur atom, $q_{\mathrm{S}}$, the $\mathrm{C}_{4 a}$ atom, $q_{\mathrm{C}_{4 a}}$, the $\mathrm{C}_{6 a}$ atom, $q_{\mathrm{C}_{6 a}}$, the dipole moment, $\mu$, the bond distances, $d$, and the bond angle, $a$. The calculated and experimental data for DBT and thiophene are reported for comparison.

\begin{tabular}{|c|c|c|c|c|c|c|c|}
\hline & $q_{\mathrm{S}}\left(\left|e^{-}\right|\right)$ & $q_{\mathrm{C}_{4 a}}\left(\left|e^{-}\right|\right)$ & $q_{\mathrm{C}_{6 a}}\left(\left|e^{-}\right|\right)$ & $\mu(\mathrm{D})$ & $d_{\mathrm{S}-\mathrm{C}_{4 a}}(\AA)$ & $d_{\mathrm{S}^{-\mathrm{C}_{6 a}}}(\AA)$ & $a_{\mathrm{C}_{4 a}-\mathrm{S}-\mathrm{C}_{6 a}}\left({ }^{\circ}\right)$ \\
\hline Thiophene & & & & 0.515 & 1.7329 & & 91.50 \\
\hline Thiophene (exp.) & & & & $0.55^{\mathrm{c}}$ & $1.7140^{\mathrm{d}}$ & & $92.17^{\mathrm{d}}$ \\
\hline DBT & -0.161 & 0.106 & & 0.793 & 1.7657 & & 90.96 \\
\hline DBT (exp.) & & & & $0.789^{\mathrm{a}}$ & $1.7345^{\mathrm{b}}$ & & $91.51^{\mathrm{b}}$ \\
\hline 3-MDBT & -0.151 & 0.147 & 0.063 & 0.993 & 1.7660 & 1.7663 & 90.97 \\
\hline 4-MDBT & -0.124 & -0.249 & 0.227 & 0.480 & 1.7689 & 1.7652 & 90.99 \\
\hline 3,6-DMDBT & -0.120 & 0.231 & -0.228 & 0.507 & 1.7654 & 1.7694 & 90.99 \\
\hline 1,3,6-TMDBT & -0.122 & 0.296 & -0.256 & 0.772 & 1.7610 & 1.7627 & 90.97 \\
\hline 2,3,6-TMDBT & -0.116 & 0.215 & -0.274 & 0.936 & 1.7661 & 17692 & 90.89 \\
\hline 3,7-DMDBT & -0.141 & 0.104 & & 0.822 & 1.7666 & & 90.98 \\
\hline 1,3,7-TMDBT & -0.129 & 0.182 & 0.072 & 1.093 & 1.7624 & 1.7601 & 90.94 \\
\hline 2,3,7-TMDBT & -0.119 & 0.065 & 0.075 & 1.126 & 1.7672 & 1.7665 & 90.87 \\
\hline 4,6-DMDBT & -0.148 & -0.052 & & 0.024 & 1.7681 & & 91.03 \\
\hline 1,4,6-TMDBT & -0.129 & 0.022 & -0.112 & 0.334 & 1.7638 & 1.7613 & 91.01 \\
\hline 2,4,6-TMDBT & -0.114 & -0.054 & -0.162 & 0.441 & 1.7684 & 1.7677 & 90.94 \\
\hline 3,4,6-TMDBT & -0.131 & -0.029 & -0.087 & 0.558 & 1.7704 & 1.7687 & 91.10 \\
\hline 1,4,7-TMDBT & -0.125 & -0.080 & 0.145 & 0.773 & 1.7652 & 1.7587 & 90.97 \\
\hline 2,4,7-TMDBT & -0.126 & -0.255 & 0.204 & 0.673 & 1.7695 & 1.7650 & 90.90 \\
\hline 3,4,7-TMDBT & -0.134 & -0.159 & 0.178 & 0.460 & 1.7743 & 1.7648 & 91.14 \\
\hline
\end{tabular}

${ }^{\mathrm{a}}[26]$.

${ }^{\mathrm{b}}[27]$.

${ }^{c}[28]$.

\section{Results and Discussion}

We obtain the most stable conformations and compare the results with available experimental data. The partial charges are evaluated using the geometry of the most stable conformations. The optimized structures of all alkyl derivatives studied show similar bond distances $\left(\mathrm{S}-\mathrm{C}_{4 a}, \mathrm{~S}-\mathrm{C}_{6 a}\right)$ and bond angles $\left(\mathrm{C}_{4 a}-\mathrm{S}-\mathrm{C}_{6 a}\right)$ to the corresponding values for the structure of DBT. We report the optimized bond lengths and bond angles near the sulfur atom using the B3LYP/6-311+G(d,p) level of theory in Table 1 . We report the optimized and experimental values for DBT and thiophene for comparison. The optimized values show absolute errors up to $1.80 \%$ in bond distances and $0.73 \%$ in bond angles. No experimental values were found for the alkyl derivatives studied in this work. The optimized values for the series of molecules studied show maximum deviations from the DBT values in 3,4,7-TMDBT for both bond distance (+0.0086 $\AA$ ) and bond angle $\left(+0.20^{\circ}\right)$. During desulfurization processes, organosulfur molecules first are adsorbed physically through the sulfur atom to the catalytic surface. The adsorbed states show weaker C-S bonds compared to the free molecules, which facilitates the extraction of the sulfur atom through the chemisorption process at the catalytic surface [10-12]. Larger C-S bonds in the free states develop weaker bonds in the adsorbed states. The differences in $\mathrm{C}-\mathrm{S}$ bond distances among the studied molecules do not explain the differences in experimental reactivity of these molecules, probably because all these molecules have the same distribution of functional groups around the sulfur atom.

We also obtain the electrostatic properties of the molecules using the same level of theory; those electrostatic properties are the base for the reactivity calculations. The charges of the studied molecules show differences up to 0.047 $\left|e^{-}\right|$in the sulfur atom compared to the DBT value (Table 1 ). The atomic charges of $\mathrm{C}_{4 a}$ and $\mathrm{C}_{6 a}$ are also reported in Table 1 because substitutions in the meta position $\left(\mathrm{C}_{1}\right.$ and $\left.\mathrm{C}_{3}\right)$ can reduce the charge of $\mathrm{C}_{4 a}$ making the lone pairs of the sulfur atom less reactive, while a substitution in the para position can increase the atomic charge of $\mathrm{C}_{4 a}$, making the sulfur atom more reactive. The lower value in the sulfur charges compared to DBT may contribute to the lower reactivity that is generally shown by the studied molecules compared with the reactivity of DBT. Interestingly, the alkyl derivative with the highest sulfur charge is the 4,6-DMDBT, one of the molecules with the lowest reactivity, but its low reactivity can be explained in terms of the level of hindrance of the sulfur atom. For the series of molecules $\{x, 4,7$-TMDBT, $(x=1,2,3)\}$, the charge of the sulfur atom and the experimental reactivity do not follow any relationship. The charge of $\mathrm{C}_{4 a}$ only predicts which molecule will be the most reactive in the series $\{x, 4,7-$ TMDBT, $(x=1,2,3)\}$ but not for the other two compounds. For all series of trimethyl derivatives studied, the charge of $\mathrm{C}_{4 a}$ predicts that methyl substitutions on $\mathrm{C}_{2}$ will be more reactive than their corresponding dimethyl derivatives. 
TABLE 2: Reactivity indexes for DBT and its methyl, dimethyl, and trimethyl derivatives, calculated using the DFT, the B3LYP/6-311+G(d,p) basis set, and the Merz-Kollman population analysis [20]: condensed Fukui functions for electrophilic attacks $\left(f_{\mathrm{S}}^{-}\right)$, vertical ionization potential (IP), vertical electron affinity (EA), global softness (S), and condensed atomic softness $\left(s_{\mathrm{S}}^{-}\right)$at sulfur atom. Calculated and experimental data for thiophene and DBT are reported for comparison.

\begin{tabular}{|c|c|c|c|c|c|c|}
\hline & $f_{\mathrm{S}}^{-}$ & IP (a.u.) & EA (a.u.) & $\chi$ (a.u.) & $\mathrm{S}\left(\right.$ a.u. $\left.^{-1}\right)$ & $s_{\mathrm{S}}^{-}\left(\right.$a.u. $\left.^{-1}\right)$ \\
\hline Thiophene & & 0.329 & -0.047 & & & \\
\hline Thiophene (exp.) & & $0.329^{\mathrm{b}}$ & $-0.042^{\mathrm{c}}$ & & & \\
\hline DBT & -0.347 & 0.286 & -0.005 & 0.141 & 3.426 & -1.189 \\
\hline DBT (exp.) & & $0.291^{\mathrm{a}}$ & & & & \\
\hline 3-MDBT & -0.342 & 0.283 & -0.008 & 0.137 & 3.433 & -1.175 \\
\hline 4-MDBT & -0.311 & 0.282 & -0.007 & 0.138 & 3.455 & -1.074 \\
\hline 3,6-DMDBT & -0.306 & 0.279 & -0.010 & 0.135 & 3.462 & -1.061 \\
\hline 1,3,6-TMDBT & -0.295 & 0.274 & -0.013 & 0.131 & 3.486 & -1.027 \\
\hline 2,3,6-TMDBT & -0.288 & 0.273 & -0.010 & 0.131 & 3.526 & -1.017 \\
\hline 3,7-DMDBT & -0.306 & 0.280 & -0.011 & 0.134 & 3.441 & -1.164 \\
\hline 1,3,7-TMDBT & -0.278 & 0.274 & -0.014 & 0.130 & 3.471 & -0.965 \\
\hline 2,3,7-TMDBT & -0.265 & 0.273 & -0.011 & 0.131 & 3.516 & -0.932 \\
\hline 4,6-DMDBT & -0.305 & 0.279 & -0.009 & 0.135 & 3.480 & -1.062 \\
\hline 1,4,6-TMDBT & -0.271 & 0.273 & -0.011 & 0.131 & 3.516 & -0.954 \\
\hline 2,4,6-TMDBT & -0.279 & 0.273 & -0.009 & 0.132 & 3.547 & -0.988 \\
\hline 3,4,6-TMDBT & -0.342 & 0.275 & -0.011 & 0.132 & 3.495 & -1.196 \\
\hline 1,4,7-TMDBT & -0.273 & 0.273 & -0.013 & 0.130 & 3.499 & -0.956 \\
\hline 2,4,7-TMDBT & -0.306 & 0.273 & -0.010 & 0.132 & 3.526 & -1.079 \\
\hline 3,4,7-TMDBT & -0.262 & 0.273 & -0.012 & 0.131 & 3.512 & -0.921 \\
\hline
\end{tabular}

a $[30]$.

${ }^{b}$ [31].

${ }^{c}[32]$.

The effect of one methyl derivative on the dipole moment of the DBT molecule is affected by its position; substitutions on $\mathrm{C}_{3}(3-\mathrm{MDBT})$ increase the dipole moment compared to DBT, while substitutions on $\mathrm{C}_{4}$ decrease the dipole moment value (4-MDBT). The dipole moment values for all molecules studied are reported in Table 1 . We also report the calculated and experimental values for DBT and thiophene in Table 1 for comparison. We find errors in the calculated values up to $6.36 \%$ from the experimental results. The effect of adding a second and third methyl group on the dipole moment value is illustrated in Figure 2. For dimethyl derivatives, the highest dipole moment is achieved in the 3,7-DMDBT and the lowest value is present in 4,6-DMDBT. Clearly the positions of the second methyl derivative also affect considerably the dipole moment value, showing the highest effect in 4,6-DMDBT; the addition of two methyl groups considerably reduces the dipole moment to very low values (compared to DBT), which in part explains the difficulties to desulfurate this molecule. The slightly higher dipole moment value present in 3,7DMDBT (compared to DBT) shows a lower value than 3MDBT, indicating a contribution elimination effect, because both substituents are located in different aromatic rings but symmetrically located with respect to the sulfur atom. For all series of compounds, the effect of a third substituent in $\mathrm{C}_{1}$ or $\mathrm{C}_{2}$ of the first aromatic ring has a positive effect on the dipole moment value if the values are compared to their correspondent dimethyl compounds. The highest dipole moment value corresponds to the molecule 2,3,7-TMDBT, where we found two effects; the first is due to the contribution elimination effect, due to substitutions on $\mathrm{C}_{3}$ and $\mathrm{C}_{7}$, which, as seen on the corresponding dimethyl molecule, only increases slightly the value of the dipole moment; the second effect is due to the substitution on $\mathrm{C}_{2}$ which is very similar to the effect found with only one substitution on $\mathrm{C}_{3}$. For substitutions on $\mathrm{C}_{3}$ (3,4,7-TMDBT and 3,4,6-TMDBT), we found a mixed behavior; in 3,4,7-TMDBT the dipole moment value slightly decreases (compared to its correspondent dimethyl molecule) and in 3,4,6-TMDBT the dipole moment value considerably increases. This can also be explained as the contribution elimination effect (3,4,7-TMDBT) and the effect of only one methyl on $\mathrm{C}_{3}(3,4,6$-TMDBT).

The reactivity descriptors $\chi$ and $S$ need energetic information (vertical IP and EA) of the molecules. To our knowledge there is no information published about these properties for the alkyl derivatives of DBT. We calculate the vertical IP and EA with the same level of theory as the geometry optimizations and report them in Table 2. We also report the calculated and the available experimental data for thiophene and DBT in Table 2. For thiophene, the calculated value for vertical IP reproduces the experimental result, while the calculated value for vertical EA shows an absolute error of $11.9 \%$. For DBT, the calculated value for IP shows an absolute error of $1.7 \%$. For the calculation of $\chi$ and $\mathrm{S}$, the high error in the calculations of EA is compensated by its low magnitude. The EA value is added or rested from the IP value to calculate $\chi$ and S (see (2) and (3)). The magnitudes of IP are between 20 


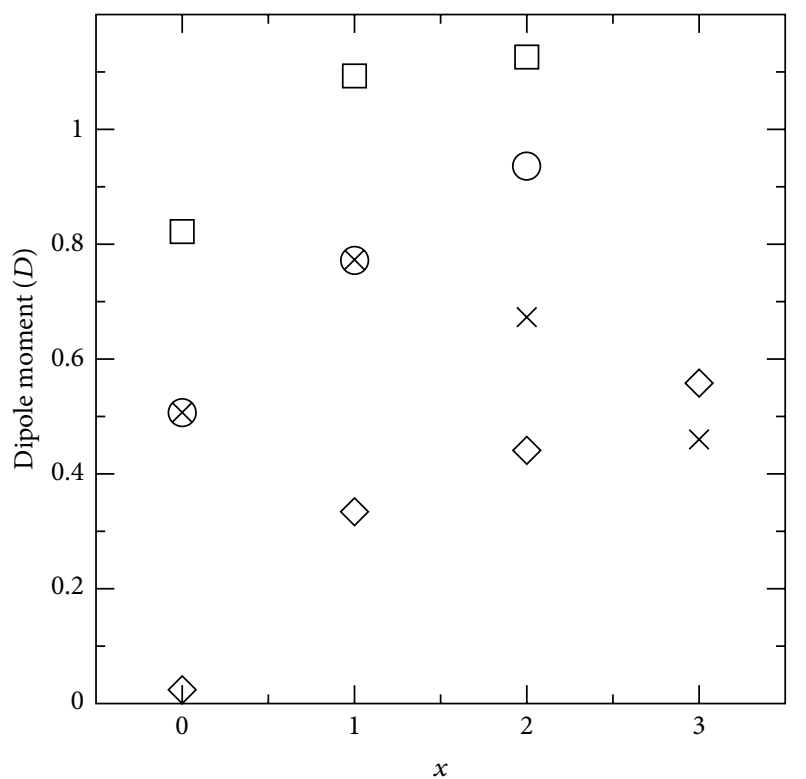

Figure 2: Dipole moment as a function of the position of the third methyl $\left(\mathrm{C}_{x}\right)$ in trimethyldibenzothiophene and its corresponding dimethyldibenzothiophene $(x=0)$. Crosses represent the series of molecules $\{x, 4,7-T M D B T,(x=1,2,3)\}$, diamonds represent $\{x, 4,6$ $\operatorname{TMDBT},(x=1,2,3)\}$, squares represent $\{x, 3,7$-TMDBT, $(x=1,2)\}$, and circles represent $\{x, 3,6$-TMDBT, $(x=1,2)\}$.

and 35 times higher than the magnitudes of EA for all alkyl derivatives studied. Then, the high errors in EA have a low impact on the final computation of $\chi$ and $S$ but also make the computed values of $\chi$ and $S$ very close and more difficult to be compared as reactivity descriptors. We report the calculated values of $\chi$ and $S$ in Table 2. We calculate the condensed Fukui functions $f_{\mathrm{S}}^{-}$and $s_{\mathrm{S}}^{-}$using (1) and (4), respectively, and also report the calculated values in Table 2. The negative values found in the Fukui function reflect the loss of charge in the sulfur atom after the electrophilic attack. Similar results have been found in the thiophene molecule [17]. As expected, the global descriptors do not have a direct relationship with the experimental available data for reactivity [3], and as the tendency of the charge of $\mathrm{C}_{4 a}$, they only predict that, for trimethyl derivatives, the substitution on the $\mathrm{C}_{2}$ will produce higher reactivities compared to their corresponding dimethyl derivatives. Figure 3 shows the reactivity descriptors $s_{S}^{-}$as a function of the natural logarithm of the relative activity for the desulfurization process for the available alkyl derivatives [3]. We calculate the experimental relative activity using the highest reported value [3], which corresponds to the activity of 1,3,7-trimethyldibenzothiophene. For the series of molecules $\{x, 4,7$-TMDBT, $(x=1,2,3)\}$ and 4-MDBT, which show the same level of hindrance in the sulfur atom, a direct relationship between $s_{\mathrm{S}}^{-}$and the relative reactivity is found, showing that the molecule 2,4,7-TMDBT is as reactive as 4-MDBT, in direct concordance with the experimental data, which classifies these two molecules with moderate reactivity [3]. The series of compounds $\{4,6$-DMDBT, 1,4,6TMDBT, and 3,4,6-TMDBT $\}$ show similar experimental reactivity and the same level of hindrance for the sulfur atom,

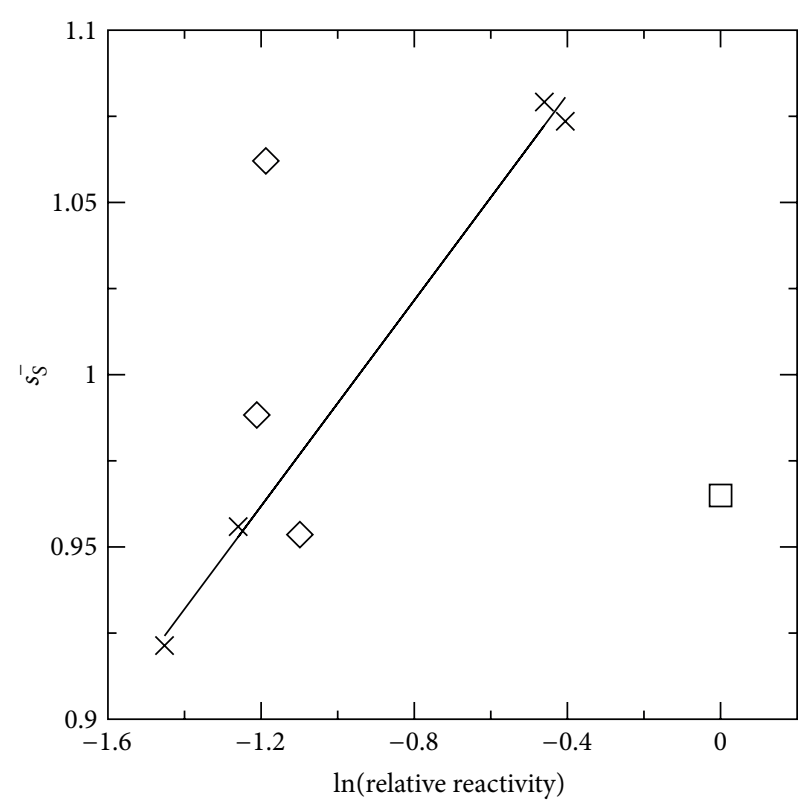

FIGURE 3: Absolute condensed atomic softness $\left(s_{\mathrm{S}}^{-}\right)$versus natural logarithm of the relative reactivity. Crosses represent the series of molecules $\{x, 4,7$-TMDBT, $(x=1,2,3)\}$ and 4-MDBT, diamonds represent $\{4,6$-DMDBT, 1,4,6-TMDBT, and 2,4,6-TMDBT $\}$, and the square represents the molecule 1,3,7-TMDBT. Relative reactivity represents experimental data from [3]. The line represents a linear regression of the plotted data for the crosses.

probably indicating that bypassing the hindrance of the sulfur atom dominates any desulfurization process in this series of molecules. The only molecule studied experimentally that does not show any level of hindrance is 1,3,7-TMDBT; it shows the highest experimental reactivity, but the $s_{\mathrm{S}}^{-}$value classifies this molecule as a compound with intermediate reactivity, which probably indicates that other factors affect the experimental reactivity of this molecule.

The MEP mapped to the electronic density isosurface is commonly used to predict the reactive sites in a molecule $[33,34]$, especially for the electrophilic attack [35]. Table 3 shows the MEPs of all molecules studied are mapped to the electronic density surface (isosurface value of $0.05 \mathrm{e}^{-} / \mathrm{Bohr}^{3}$ ). The MEP of DBT shows three possible adsorption sites; one is located at the sulfur atom; two are located at carbon atoms in the aromatic rings $\left(\mathrm{C}_{2}\right.$ and $\left.\mathrm{C}_{8}\right)$, which is in agreement with experimental results for adsorption of DBT on catalytic surfaces $[36,37]$. The sites located at the aromatic rings compete with the sulfur atom during adsorption processes and result in adsorbed states with different orientation to the surface. The orientation is flat when the molecule is adsorbed through the aromatic ring and perpendicular when adsorbed through the sulfur atom [11]. The perpendicular adsorption through the sulfur atom is needed for the direct desulfurization processes $[1,2]$. Due to the steric effect on the sulfur atom, the desulfurization process of the studied molecules occurs through intermediate hydrogenation processes of the aromatic rings [1] or direct desulfurization at edges or vacancies in the catalysts [11]. Hydrogenation 
TABLE 3: Molecular electrostatic potential mapped at the electronic density isosurface for DBT and its methyl, dimethyl, and trimethyl derivatives. Blue areas represent regions where a proton would feel repulsion while in red areas a proton would feel attraction. DBT, 3-MDBT, and 4-MDBT are also plotted for comparison.

\begin{tabular}{|c|c|c|c|c|}
\hline & $x, 3,6$-TMDBT & $x, 3,7$-TMDBT & $x, 4,6$-TMDBT & $x, 4,7-\mathrm{TMDBT}$ \\
\hline \multicolumn{5}{|c|}{$\begin{array}{l}\text { DMDBT } \\
x=0\end{array}$} \\
\hline \multicolumn{5}{|l|}{$x=1$} \\
\hline \multicolumn{5}{|l|}{$x=2$} \\
\hline$x=3$ & & & & \\
\hline
\end{tabular}

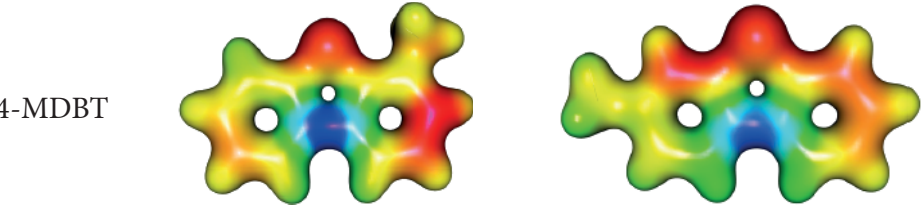

3-MDBT

DBT

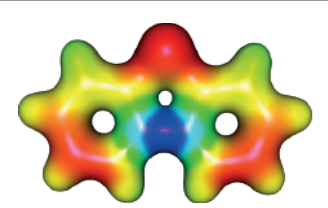

processes occur through adsorptions using the carbon atoms in the aromatic rings as active sites.

The addition of one methyl group to the DBT structure reduces the number of adsorption sites; if the methyl is located at the $\mathrm{C}_{4}$ atom (4-MDBT), the adsorption site located at $\mathrm{C}_{9}$ disappears and the site located at $\mathrm{C}_{2}$ moves to the area located at the bond $\mathrm{C}_{2}-\mathrm{C}_{3}$, while if the methyl group is located at the $\mathrm{C}_{3}$ atom (3-MDBT), the adsorption site located at $\mathrm{C}_{2}$ and $\mathrm{C}_{8}$ disappears and the adsorption site located at the sulfur atom grows to also cover the bonds $\mathrm{C}_{4}-\mathrm{C}_{4 a}$ and $\mathrm{C}_{6}-\mathrm{C}_{6 a}$. Therefore, the methylation at the $\mathrm{C}_{3}$ atom will facilitate the desulfurization process because it will eliminate the competitive adsorption through the carbon rings, while leaving the access to the sulfur atom free.

The addition of a second methyl to form 3,6-DMDBT and 3,7-DMDBT does not affect the distribution of adsorption sites. 3,6-DMDBT has the same distribution of adsorption sites as 4-MDBT, and 3,7-DMDBT has the same distribution as 3-MDBT. 4,7-DMDBT has the same structure as 3,6DMDBT. 4,6-DMDBT increases the number of adsorption sites to three and has a similar distribution of adsorption sites as the DBT molecule. A third methyl in the series of molecules $\{x, 3,6$-TMDBT, $(x=1,2)\}$ and $\{x, 3,7-T M D B T$, $(x=1,2)\}$ does not affect the distribution of adsorption sites, and they have the same distribution of sites as their corresponding dimethyl derivatives. For the series of molecules $\{x, 4,6$-TMDBT, $(x=1,2,3)\}$, only when the third methyl is located at the $\mathrm{C}_{2}$ atom $(2,4,6$-TMDBT), the number of adsorption locations reduces to two sites, but the capacity of adsorption of the sulfur atom site decreases considerably. For the series of molecules $\{x, 4,7$-TMDBT, $(x=1,2,3)\}$, $1,4,7-$ TMDBT shows an adsorption site at the $\mathrm{C}_{2}-\mathrm{C}_{3}$ bond with no methyl substitutes in the aromatic ring blocking its activity. 1,4,7-TMDBT and 3,4,7-TMDBT have an active site for adsorption at the $\mathrm{C}_{8}$ carbon with one methyl substitute blocking its activity. 2,4,7-TMDBT has an adsorption site 
at the $\mathrm{C}_{3}$ atom, but the activity of $\mathrm{C}_{3}$ is blocked by two methyl substitutes. 2,4,7-TMDBT also has an active site on $\mathrm{C}_{6}$, but with $\mathrm{C}_{6}$ being close to the highest active site $(\mathrm{S})$ within 2,4,7-TMDBT, its activity is lowered. Therefore, the molecule that shows less competitive activity to the sulfur atom is 2,4,7-TMDBT, because it shows one competitive site and the activity of this site is more hindered than the possible activity of the sulfur atom.

\section{Conclusions}

We calculate the structural, electrostatic, and energetic properties of DBT and its methyl, dimethyl, and trimethyl derivatives using DFT calculations at the B3LYP/6-311+G(d,p) level of theory.

The addition of methyl groups to the DBT structure modifies the dipole moment of the alkyl derivatives. With the dipole moment being an important parameter in the adsorption processes, we find that substitutions away from the sulfur atom $\left(\mathrm{C}_{2}\right.$ or $\left.\mathrm{C}_{3}\right)$ increase the dipole moment. If the methylation produces symmetric substitutions in both rings (3,7-DMDBT), the increase in magnitude of the dipole moment is low due to a blocking effect. Substitutions near the sulfur atom $\left(\mathrm{C}_{4}\right.$ or $\left.\mathrm{C}_{6}\right)$ decrease the dipole moment due to a blocking effect of the methyls on the sulfur charge, which has a positive contribution to the dipole moment.

We also predict the reactivity trend in the series of molecules, which can be explained using $s_{S}^{-}$. The results for $s_{S}^{-}$show that the reactivity is linked to the activity of the sulfur atom for the series of molecules $\{x, 4,7-T M D B T,(x=$ $1,2,3)\}$ and show the trend of 3,4,7-TMDBT $<1,4,7-$ TMDBT $<2,4,7-T M D B T$, in agreement with the experimental results for desulfurization reactions. The reactivity trend is the result of the position of the third methyl substitute, because that position activates/deactivates the $\mathrm{C}_{4 a}-\mathrm{S}$ bond. For the series of molecules $\{x, 4,6$-TMDBT, $(x=1,2,3)\}, s_{\mathrm{S}}^{-}$does not predict the experimental behavior, probably due to the high level of hindrance of the sulfur atom.

The MEP of DBT and its alkyl derivatives show an adsorption site located at the sulfur atom. There are other adsorption sites located at the carbons or the bonds forming the aromatic rings that can compete with the sulfur atom in adsorption processes. Only the structures of 3-MDBT and its alkyl derivatives reduce the number of adsorption sites to one located at the area near the sulfur atom and its neighbor bonds and carbon atoms. The 4,6-DMDBT and its alkyl derivatives $\{x, 4,6$-TMDBT, $(x=1,2,3)\}$ show competitive adsorption sites located in the aromatic rings, which enhances the problem to desulfurate these molecules. Among the series $\{x, 4,7-T M D B T,(x=1,2,3)\}$, only the $2,4,7-$ TMDBT molecule shows one active site near the sulfur atom, explaining its high reactivity compared to the other molecules of this series of compounds.

\section{Conflict of Interests}

The authors declare that there is no conflict of interests regarding the publication of this paper.

\section{Acknowledgments}

Jose Luis Rivera thanks Universidad Michoacana de San Nicolás de Hidalgo (México) and CONACYT (México) for support under Grant no. 134508 for young researchers. Pedro Navarro-Santos also thanks CONACYT for a postdoctoral fellowship (170026). Roberto Guerra-Gonzalez thank PROMEP (Mexico) for financial assistance under Grant no. UMSNH-EXB-223.

\section{References}

[1] R. Prins, M. Egorova, A. Röthlisberger, Y. Zhao, N. Sivasankar, and P. Kukula, "Mechanisms of hydrodesulfurization and hydrodenitrogenation," Catalysis Today, vol. 111, no. 1-2, pp. 8493, 2006.

[2] H. Yang, J. Chen, Y. Briker, R. Szynkarczuk, and Z. Ring, "Effect of nitrogen removal from light cycle oil on the hydrodesulphurization of dibenzothiophene, 4-methyldibenzothiophene and 4,6-dimethyldibenzothiophene," Catalysis Today, vol. 109, no. 14, pp. 16-23, 2005.

[3] H. Yang, J. Chen, C. Fairbridge, Y. Briker, Y. J. Zhu, and Z. Ring, "Inhibition of nitrogen compounds on the hydrodesulfurization of substituted dibenzothiophenes in light cycle oil," Fuel Processing Technology, vol. 85, no. 12, pp. 1415-1429, 2004.

[4] J. Chen, H. Yang, and Z. Ring, "HDS kinetics study of dibenzothiophenic compounds in LCO," Catalysis Today, vol. 98, no. 1-2, pp. 227-233, 2004.

[5] X. Ma, K. Sakanishi, and I. Mochida, "Hydrodesulfurization reactivities of various sulfur compounds in vacuum gas oil," Industrial and Engineering Chemistry Research, vol. 35, no. 8, pp. 2487-2494, 1996.

[6] I. García-Cruz, D. Valencia, T. Klimova et al., "Proton affinity of S-containing aromatic compounds: implications for crude oil hydrodesulfurization," Journal of Molecular Catalysis A, vol. 281, no. 1-2, pp. 79-84, 2008.

[7] F. Juárez-Guerra, J. L. Rivera, A. Zúñiga-Moreno, L. A. GaliciaLuna, J. L. Rico, and J. Lara, "Molecular modeling of thiophene in the vapor-liquid equilibrium," Separation Science and Technology, vol. 41, no. 2, pp. 261-281, 2006.

[8] B. Gómez and J. M. Martińez-Magadán, "A theoretical study of dibenzothiophene absorbed on open-ended carbon nanotubes," Journal of Physical Chemistry B, vol. 109, no. 31, pp. 14868-14875, 2005.

[9] X. Rozanska, R. A. van Santen, F. Hutschka, and J. Hafner, "A periodic density functional theory study of thiophenic derivative cracking catalyzed by mordenite," Journal of Catalysis, vol. 215, no. 1, pp. 20-29, 2003.

[10] H. Yang, C. Fairbridge, and Z. Ring, "Adsorption of dibenzothiophene derivatives over a $\mathrm{MoS}_{2}$ nanocluster-a density functional theory study of structure-reactivity relations," Energy and Fuels, vol. 17, no. 2, pp. 387-398, 2003.

[11] S. Cristol, J. F. Paul, E. Payen, D. Bougeard, F. Hutschka, and S. Clémendot, "DBT derivatives adsorption over molybdenum sulfide catalysts: a theoretical study," Journal of Catalysis, vol. 224, no. 1, pp. 138-147, 2004.

[12] P. Raybaud, J. Hafner, G. Kresse, and H. Toulhoat, "Adsorption of thiophene on the catalytically active surface of $\mathrm{MoS}_{2}$ : an $\mathrm{Ab}$ Initio local-density-functional study," Physical Review Letters, vol. 80, no. 7, pp. 1481-1484, 1998. 
[13] J. L. Rivera, J. L. Rico, and F. W. Starr, "Interaction of water with cap-ended defective and nondefective small carbon nanotubes," Journal of Physical Chemistry C, vol. 111, no. 51, pp. 18899-18905, 2007.

[14] C. Makedonas and C. A. Mitsopoulou, "An investigation of the reactivity of [(diimine)(dithiolato)M] complexes using the Fukui functions concept," European Journal of Inorganic Chemistry, no. 3, pp. 590-598, 2006.

[15] R. Meza, B. Gordillo, and M. Galván, "Local HSAB principle in the conjugate addition of p-Substituted thiophenols to cyclohexenone," International Journal of Quantum Chemistry, vol. 104, no. 1, pp. 29-37, 2005.

[16] C. Martinez, J. L. Rivera, R. Herrera et al., "Evaluation of the chemical reactivity in lignin precursors using the Fukui function," Journal of Molecular Modeling, vol. 14, no. 2, pp. 7781, 2008.

[17] R. Salcedo, A. Martínez, and L. E. Sansores, "Five and nine membered (heteronines) heterocyclic molecules. Theoretical approach," Tetrahedron, vol. 57, no. 42, pp. 8759-8765, 2001.

[18] M. J. Frisch, G. W. Trucks, H. B. Schlegel et al., "Gaussian 03, Revision C. 02," Gaussian, Inc., Wallingford, Conn, USA, 2004.

[19] T. C. Dinadayalane, G. Narahari-Sastry, and J. Leszczynski, "Comprehensive theoretical study towards the accurate proton affinity values of naturally occurring amino acids," International Journal of Quantum Chemistry, vol. 106, no. 14, pp. 2920-2933, 2006.

[20] B. H. Besler, K. M. Merz Jr., and P. A. Kollman, "Atomic charges derived from semiempirical methods," Journal of Computational Chemistry, vol. 11, pp. 431-439, 1990.

[21] R. G. Parr and W. Yang, "Density functional approach to the frontier-electron theory of chemical reactivity," Journal of the American Chemical Society, vol. 106, no. 14, pp. 4049-4050, 1984.

[22] R. G. Parr and W. Yang, Density Functional Theory of Atoms and Molecules, Oxford University Press, New York, NY, USA, 1989.

[23] H. Chermette, "Chemical reactivity indexes in density functional theory," Journal of Computational Chemistry, vol. 20, no. 1, pp. 129-154, 1999.

[24] P. Geerlings, F. de Proft, and W. Langenaeker, "Conceptual density functional theory," Chemical Reviews, vol. 103, no. 5, pp. 1793-1873, 2003.

[25] R. G. Pearson, "Absolute electronegativity and hardness: application to inorganic chemistry," Inorganic Chemistry, vol. 27, no. 4, pp. 734-740, 1988.

[26] T. Nagai, Y. Koga, H. Takahashi, and K. Higasi, "Dielectric relaxation and molecular structure. XII. Dipole moments and dielectric relaxation times of rigid molecules in solutions," Bulletin of the Chemical Society of Japan, vol. 47, pp. 1022-1023, 1974.

[27] R. M. Schaffrin and J. Trotter, "Structure of dibenzothiophen," Journal of the Chemical Society A, pp. 1561-1565, 1970.

[28] R. D. Brown, F. R. Burden, and P. D. Godfrey, "The microwave spectrum of selenophene," Journal of Molecular Spectroscopy, vol. 25, no. 4, pp. 415-421, 1968.

[29] B. Bak, D. Christensen, L. Hansen-Nygaard, and J. RastrupAndersen, "The structure of thiophene," Journal of Molecular Spectroscopy, vol. 7, no. 1-6, pp. 58-63, 1961.

[30] B. Ruscic, B. Kovac, L. Klasinc, and H. Gusten, "Photoelectron-spectroscopy of heterocycles-fluorine analogs," Zeitschrift für Naturforschung A, vol. 33, pp. 1006-1012, 1978.
[31] J. J. P. Stewart, "Optimization of parameters for semiempirical methods II. Applications," Journal of Computational Chemistry, vol. 10, pp. 221-264, 1989.

[32] E. H. van Veen, "Triplet $\pi \rightarrow \pi^{*}$ transitions in thiophene, furan and pyrrole by low-energy electron-impact spectroscopy," Chemical Physics Letters, vol. 41, no. 3, pp. 535-539, 1976.

[33] B. Galabov, P. Bobadova-Parvanova, S. Ilieva, and V. Dimitrova, "The electrostatic potential at atomic sites as a reactivity index in the hydrogen bond formation," Journal of Molecular Structure, vol. 630, no. 1-3, pp. 101-112, 2003.

[34] F. Blockhuys, C. Peten, C. van Alsenoy, and H. J. Geise, "Investigation of the reactivity of thiophene compounds towards butyllithium using the molecular electrostatic potential," Journal of Molecular Structure, vol. 445, no. 1-3, pp. 187-195, 1998.

[35] G. Náray-Szabó and G. G. Ferenczy, "Molecular electrostatics," Chemical Reviews, vol. 95, no. 4, pp. 829-847, 1995.

[36] T. Kabe, A. Ishihara, and Q. Zhang, "Deep desulfurization of light oil. Part 2: hydrodesulfurization of dibenzothiophene, 4methyldibenzothiophene and 4,6-dimethyldibenzothiophene," Applied Catalysis A, vol. 97, no. 1, pp. L1-L9, 1993.

[37] M. Seredych and T. J. Bandosz, “Template-derived mesoporous carbons with highly dispersed transition metals as media for the reactive adsorption of dibenzothiophene," Langmuir, vol. 23, no. 11, pp. 6033-6041, 2007. 

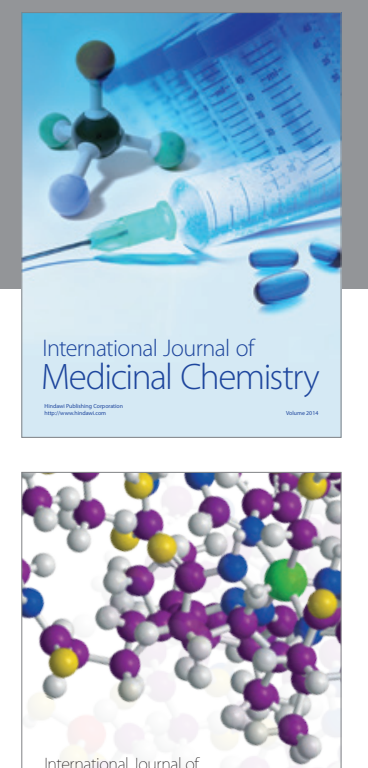

\section{Carbohydrate} Chemistry

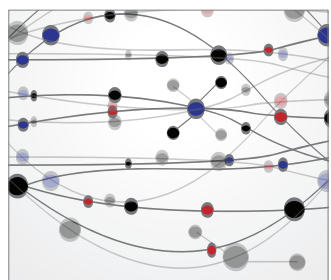

The Scientific World Journal
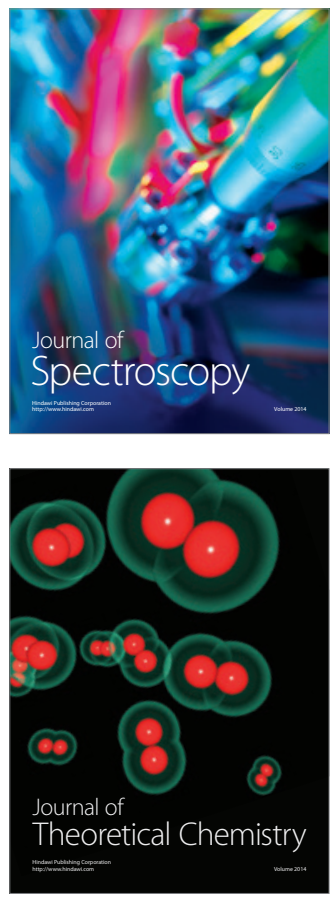
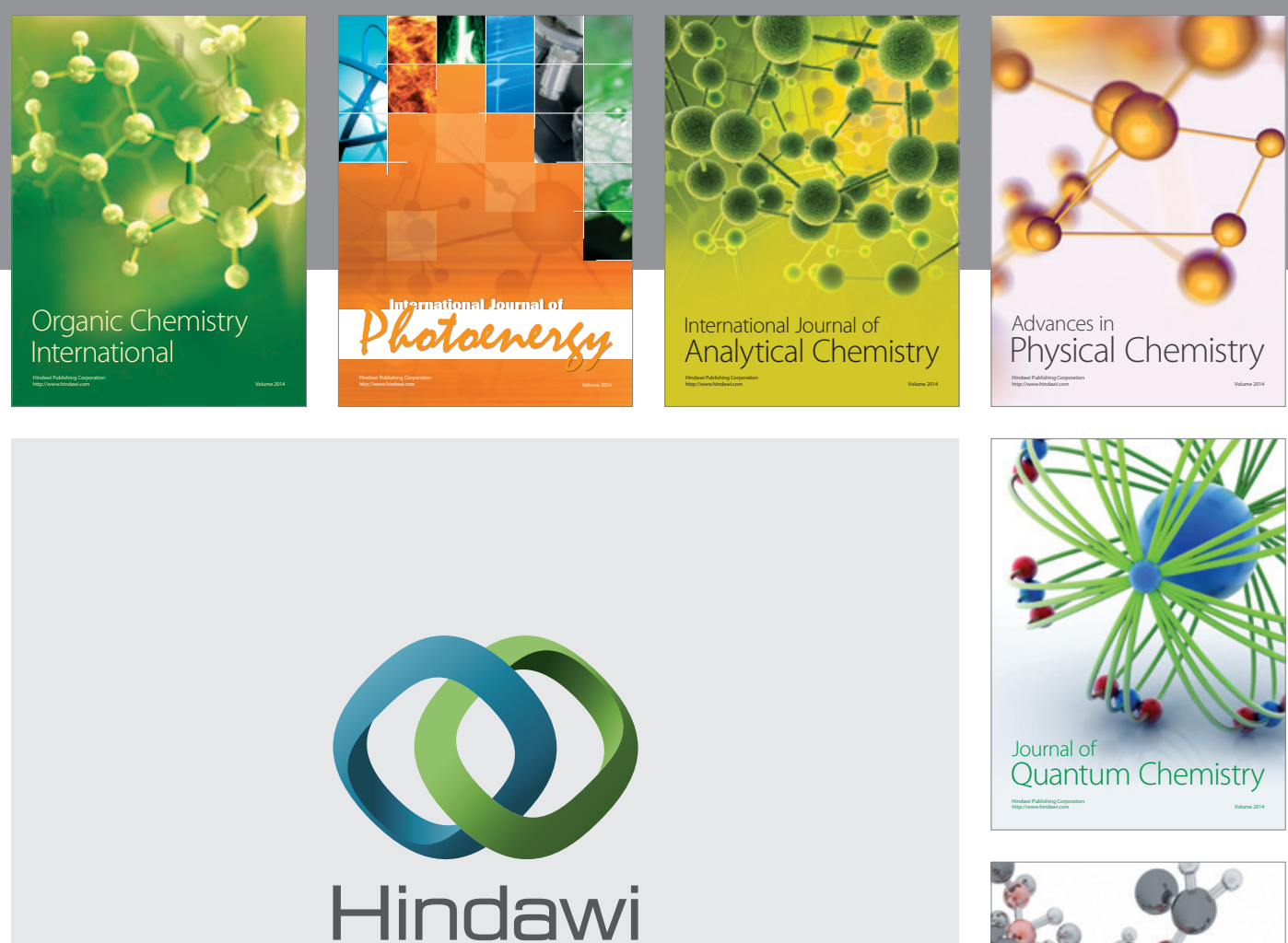

Submit your manuscripts at

http://www.hindawi.com

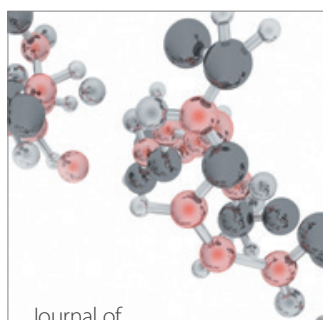

Analytical Methods

in Chemistry

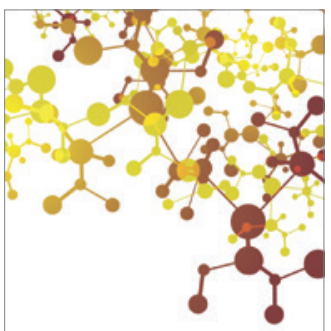

Journal of

Applied Chemistry

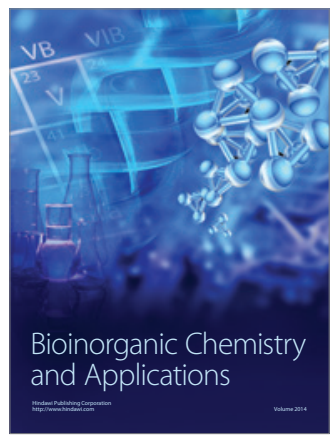

Inorganic Chemistry
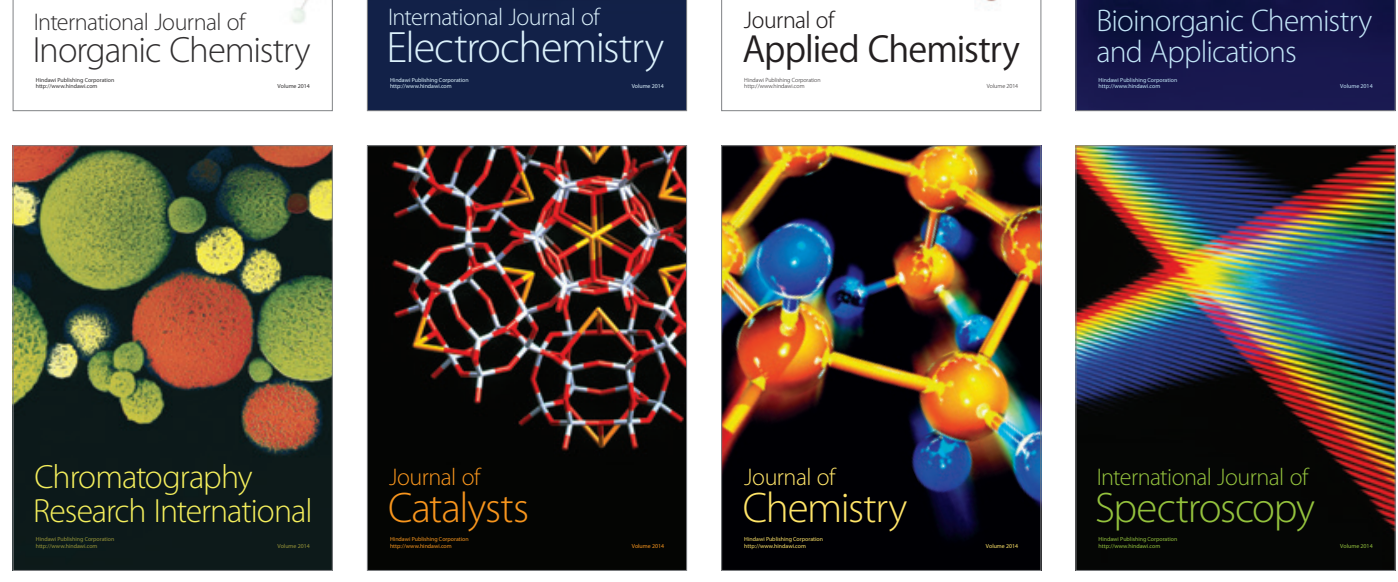